\title{
Triggers of daily life ischaemia
}

\author{
S B Freedman, C K Wong
}

\begin{abstract}
Objective-To determine the usual triggers of silent and symptomatic ischaemia. Design-Patients wore an ambulatory recorder for 48 hours. The device emitted a tone on detection of ischaemia and patients noted activities, feelings, and symptoms so that ischaemia could be attributed to one of four triggers: physical stress, mental stress, combined physical/mental stress, or no stressor.

Setting-Home environment.
\end{abstract}

Patients-Patients $(\mathrm{n}=38)$ with stable coronary disease, positive exercise electrocardiography, and ischaemic episodes on ambulatory electrocardiography.

Main outcome measure-Matching ischaemic episodes with perceived triggers.

Results-Altogether 257 ischaemic episodes (53\% silent) were documented. Triggers were: physical stress, 56\%; mental stress, 5\%; combined physical/mental stress, $8 \%$; no identifiable trigger, $31 \%$. Episodes associated with mental or no stress were more often silent $(69 \%$ and $75 \%$, respectively) than those associated with physical stress $(45 \%, p<0.01)$, while combined physical/mental stress episodes were usually symptomatic $(10 \%$ silent, p $<0.01 v$ other stressors). Although physical stress was less commonly a trigger of silent ischaemia than angina $(47 \% v 65 \%, p<0.01)$, it was still the predominant trigger of silent ischaemia. There was no identifiable trigger in $45 \%$ of silent and only $17 \%$ of anginal episodes $(p<0.01)$. Only nine silent episodes involved mental stress alone as a trigger. Conclusions-Daily life ischaemia is usually triggered by physical activity. Mental stress alone is an uncommon trigger of either silent or symptomatic ischaemia, while combined physical/mental stress is a significant but minor trigger of angina. Patients can identify a trigger in $83 \%$ of anginal episodes, compared with only half of silent ischaemic episodes.

(Heart 1998;80:489-492)

Keywords: silent ischaemia; ambulatory electrocardiography

Patients with angina pectoris readily induced by exercise stress testing have been shown by ambulatory recordings to experience frequent episodes of ischaemia during daily life, but as most of these ischemic episodes are silent, ${ }^{1-6}$ it is difficult to determine their cause. Ischaemia can be triggered in the absence of physical exertion by stimuli such as mental stress, ${ }^{278}$ smoking, ${ }^{910}$ and cold immersion, ${ }^{11}$ although in those situations ischaemia is generally milder, with less ST change and less angina, ${ }^{2}{ }^{212-14}$ and may require more sophisticated methods for diagnosis based on wall motion or perfusion abnormalities. $^{27913}$ It has been tempting to attribute many of these silent episodes to mental stress, ${ }^{15}{ }^{16}$ although the methods used in previous studies - such as structured or random prompted entry diaries-do not permit precise temporal correlations between the onset of ischaemia and what the patient is doing or thinking at the time.

In our present study, we used microprocessor technology to make a continuous analysis of the ambulatory electrocardiogram (QMED recorder).$^{17}$ This recorder can be programmed to emit a tone when ischaemia is detected, presenting a unique opportunity for patients to record their thoughts and activities during ischaemia and in the period immediately preceding it. The purpose of this study was to determine the usual triggers of myocardial ischaemia during daily life.

\section{Methods}

PROTOCOL

Sixty five patients with stable angina pectoris and a positive exercise stress test were screened for ambulatory ischaemia. All patients then underwent two days of ambulatory electrocardiography on their usual drugs. During the two days of electrocardiographic recording, patients filled in a diary as described below.

\section{Ambulatory ST monitoring and diary}

A two channel real time ambulatory recorder (QMED-TC350) ${ }^{17}$ continuously analysed the two leads which showed maximum ST depression during exercise testing (QMED Inc, Laurence Harbor, New Jersey, USA). The recorder was programmed to detect episodes of ischaemia defined as planar or downsloping ST depression of more than $0.1 \mathrm{mV}$ measured 60 $\mathrm{ms}$ after the J point, lasting for more than 40 seconds. Whenever ischaemia was detected, the recorder sounded an audible tone. Patients were not told what the significance of the tone was. They were requested to enter the time and the presence or absence of angina in a diary each time they heard a tone. They were also instructed to fill in the following items describing their thoughts, feelings, activity, and stress level at the time of the tone or in the five minutes immediately preceding it:

- activity

- thoughts

- feelings

- physical stress, graded by the patients as none, light, moderate, or vigorous (physical activity categories $\mathrm{A}, \mathrm{B}, \mathrm{C}$, and $\mathrm{D}$, respectively) 
Table 1 Triggers and heart rate at the onset of the 257 verified ambulatory ischaemic episodes (136 silent) with a matching diary entry

\begin{tabular}{|c|c|c|c|c|c|c|c|}
\hline \multirow[b]{2}{*}{ Trigger } & \multicolumn{3}{|c|}{$\begin{array}{l}\text { All ischaemic episodes } \\
(n=257)\end{array}$} & \multicolumn{2}{|c|}{$\begin{array}{l}\text { Silent episodes } \\
(n=136)\end{array}$} & \multicolumn{2}{|c|}{$\begin{array}{l}\text { Anginal episodes } \\
(n=121)\end{array}$} \\
\hline & $n$ & $\%$ Total & HR at onset & $n$ & $\%$ Total & $n$ & $\%$ Total \\
\hline Physical stress only & 143 & 56 & $91(17)^{\star}$ & 64 & 47 & 79 & $65 \ddagger$ \\
\hline Mental stress only & 13 & 5 & $85(12)$ & 9 & 7 & 4 & 3 \\
\hline Both physical stress and mental stress & 20 & 8 & $104(13) \dagger$ & 2 & 1 & 18 & $15 \ddagger$ \\
\hline Nil trigger & 81 & 31 & $82(18)$ & 61 & 45 & 20 & $17 \ddagger$ \\
\hline
\end{tabular}

${ }^{\star} \mathrm{p}<0.05 v$ nil trigger $; \mathrm{tp}<0.05 v$ all other triggers; $\neq \mathrm{p}<0.01$ anginal episodes $v$ silent episodes.

$\mathrm{HR}$ at onset, mean (SD) of heart rate at onset of ischaemia; Nil trigger, no trigger identified by patient.

- mental stress, graded by the patients on an analogue scale of 0 to 10 ( 0 , no thoughts or feelings; 1 , no perceptible mental stress; 2, minimal perceptible stress; 10, highest level of stress).

In the study design, both physical and mental stress were defined in a dichotomous manner as any stress the patient perceived, no matter how trivial. For physical stress this was any physical activity, or physical stress categories B, C, or D. For mental stress this was any thought or feeling that might denote mental stress, or a mental stress grading of 2 or more. The patients were unaware of this dichotomy. This was felt necessary because stress perception is highly subjective and it was felt that a liberal definition would ensure a high sensitivity.

The output of the ambulatory ECG recorders was verified to ensure that all episodes of ischaemia detected by the recorder represented ischaemic ST segment depression as defined above. Patient diaries were then examined to ensure that timing of patient entries matched the verified ischaemic episodes.

\section{PATIENTS}

Forty five patients had at least one verified ischaemic episode on Holter recordings, and 38 had a diary entry matching at least one recorded ischaemic episode. These 38 patients (31 men and seven women, mean (SD) age 61 (8) years) form the basis of this report. Thirty four of the 38 patients had angiographically documented coronary disease $(70 \%$ lumen diameter reduction of at least one coronary artery). The remaining four had typical angina pectoris and a positive exercise stress test. All were clinically stable, and 35 were on background antianginal treatment in addition to sublingual nitrates ( $\beta$ blocker 13 , calcium antagonist 20, long acting nitrate 30 ). Twenty one had experienced myocardial infarction (all more than three months earlier), and 20 were on the waiting list for coronary bypass surgery. All gave written consent to the study, which was approved by the institutional ethics review committee.

\section{STATISTICS}

Differences in means between groups were compared with either unpaired $t$ tests or one way analysis of variance as appropriate, with Bonferroni corrections for multiple comparisons. Differences in proportions were compared using the exact binomial conditional test. Probability ( $\mathrm{p}$ ) values of $<0.05$ (two tailed) were considered significant. Values are given as mean (SD).

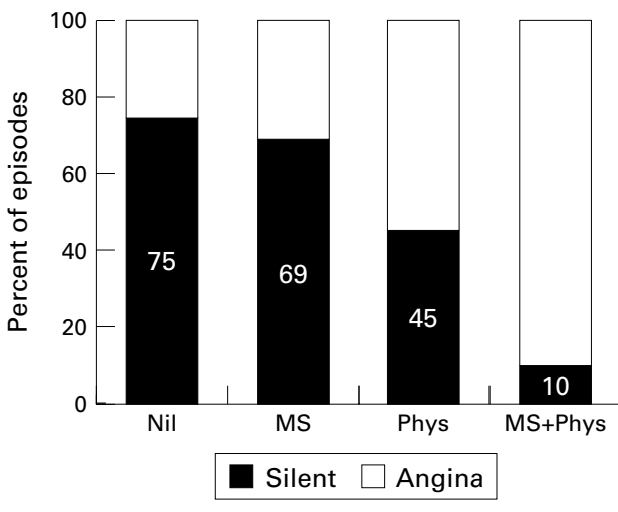

Figure 1 Percentage of ischaemic episodes that were silent (solid portion of bars and numerals) or symptomatic (open portion of bars). Episodes have been divided into four trigger categories: Nil, episodes with no identified trigger $(n=81) ; M S$, episodes attributed to mental stress alone ( $n=13)$; Phys, episodes attributed to physical stress alone $(n=143) ; M S+P h y s$, episodes attributed to combined physical and mental stress $(n=20) . p<0.01$ for comparison of Phys $v$ Nil and MS; $p<0.01$ for comparison of $M S+P h y s v$ all other categories.

\section{Results}

EXERCISE TESTING

Only 26 patients experienced angina during exercise, although all had a positive exercise ECG. The mean (SD) maximum workload was 66 (25) watts, with a mean total exercise duration of 5.2 (2.7) minutes. The mean time to 1 mm ST depression was 3.7 (2.5) minutes, and the mean heart rate at $1 \mathrm{~mm} \mathrm{ST} \mathrm{depression} \mathrm{was}$ 91 (14) beats/min.

AMBULATORY ISCHAEMIA AND DIARY

INTERROGATION

During a median of 47 hours of ambulatory recording, 257 episodes of ischaemia with a matching diary entry were documented and analysed. Patients experienced a median of four episodes during the recording, with a median duration of 19 minutes of ischaemia each day. Episodes of ischaemia were attributed to one of four trigger categories depending on their association with physical or mental stress as defined above: physical stress; mental stress; physical plus mental stress; no stressor identified.

RELATION BETWEEN ISCHAEMIC EPISODES, STRESSORS, AND SYMPTOMS

The proportion of episodes allocated to each category is shown in table 1 . Most ischaemic episodes were associated with physical stress alone $(56 \%)$, while $31 \%$ had no identified trigger. Only 13 of the 257 episodes (5\%) were associated with perceived mental stress alone, 

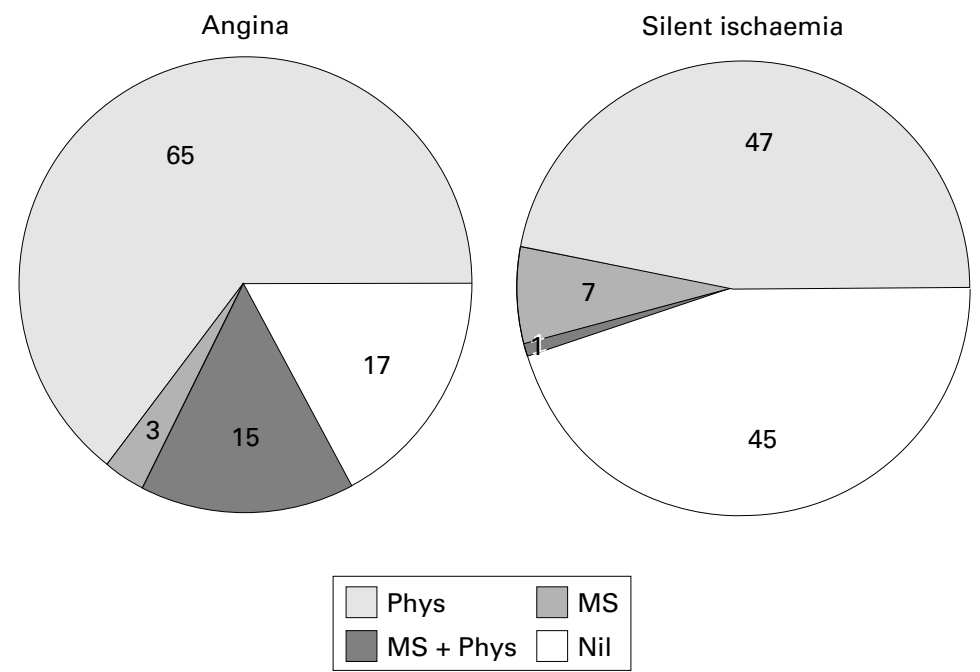

Figure 2 Breakdown of triggers as a percentage of anginal episodes $(n=136$, left $)$ and silent ischaemic episodes $(n=121$, right). Phys, episodes attributed to physical stress alone; $M S$, episodes attributed to mental stress alone; $M S+P h y s$, episodes attributed to combined physical and mental stress; Nil, episodes with no identifiable trigger.

and another $8 \%$ were associated with combined physical and mental stress.

Of the 257 ischaemic episodes, just over half (53\%) were silent. Episodes associated with mental stress, or no identified stressor, were more often silent ( $69 \%$ and $75 \%$, respectively) than episodes associated with physical stress $(45 \%$ silent, $\mathrm{p}<0.01)$, while episodes associated with both physical and mental stress were usually symptomatic ( $10 \%$ silent, $\mathrm{p}<0.01 v$ other groups) (table 1; fig 1). While physical stress was significantly less common as a trigger of silent ischaemia than angina $(47 \% v$ $65 \%, \mathrm{p}<0.01$, fig 2 ), it was still the predominant trigger of silent ischaemia. On the other hand, nearly half ( $45 \%)$ of all silent episodes had no identifiable trigger, compared with only $17 \%$ of anginal episodes ( $p<0.01$, fig 2$)$. Only $7 \%$ of all silent ischaemic episodes (nine episodes) were triggered by mental stress alone, and a further two by a combination of physical and mental stress (table 1, fig 2). Combined physical and mental stress was a small but significant trigger of angina, but was uncommon as a cause of silent ischaemia (15\% $v 1 \%, \mathrm{p}<0.01$, fig 2 ).

RELATION BETWEEN HEART RATE, GRADING OF PHYSICAL STRESS, AND ISCHAEMIA

The mean heart rate at onset of ischaemia was slightly higher in symptomatic than in silent episodes (91 (17) v 87 (18) beats/min, $\mathrm{p}=0.05)$. Overall, episodes associated with physical stress had a higher heart rate than those with no stress or mental stress, but episodes triggered by combined physical and mental stress had the highest heart rate (table 1). Further analysis of episodes associated with physical stress showed that the mean heart rate was higher with vigorous effort (101 (17) beats/min, 27 episodes) than with moderate effort (89 (16) beats/min, 36 episodes) or light effort (88 (16) beats/min, 80 episodes, $\mathrm{p}<0.01$ for both comparisons). However, the mean heart rate at the onset of ambulatory ischaemia was considerably lower than that at
$1 \mathrm{~mm} \mathrm{ST}$ depression during exercise testing (91 (14) v 109 (6) beats/min, $\mathrm{p}<0.05$ ).

\section{Discussion}

The novel application of microprocessor technology in this study provided a unique opportunity to get reliable temporal information about physical and mental activities at the exact moment of ischaemia in everyday life. In this study we showed that daily life ischaemia in patients with stable coronary disease, whether painful or silent, was usually triggered by physical activity, which is often minor. In almost one third of episodes, however, patients were unable to identify a trigger. Mental stress alone was an uncommon trigger of either silent or symptomatic ischaemia, although combined mental and physical stressors accounted for a significant though small proportion of symptomatic ischaemia.

A striking finding in this study was the difference between silent ischaemia and angina in triggers of ischaemia. In nearly half of all silent ischaemic episodes patients were unable to identify a trigger - a much greater proportion than seen with angina, where patients were able to identify a trigger in $83 \%$ of episodes. Physical stress was the trigger for most of the remaining silent ischaemic episodes, with mental stress alone responsible for a small minority, as was the case for angina.

These results indicate a lesser role of mental stress alone in daily life ischaemia than suggested by previous studies, which relied on patients keeping a continuous diary of physical and mental activity throughout the day. ${ }^{15}{ }^{16} \mathrm{We}$ did not attempt to correct for the relative duration of mental or physical stress during the day, as was done in those studies. The emphasis instead was to determine the perceived triggers of the observed silent ischaemic episodes. Given the probable wide fluctuation of stressors throughout the day, this method may be more accurate in representing what was occurring at the precise time of ischaemia and in the few minutes leading up to it, and would be less intrusive on daily life activities than continuous or prompted diary entry.

In the present study, only $53 \%$ of the ischaemic episodes were silent, less than the $70-90 \%$ reported in other studies. ${ }^{1-4}$ This may result from the methodology, as patients prompted by the tone may have reported very mild symptoms which might otherwise be ignored. In addition, we were able to analyse only those episodes with a matching diary entry. In several patients, ischaemic episodes occurred without a matching diary entry, and for these episodes we are uncertain why patients did not respond to the tone. It is likely that they were asleep, or did not hear the tone, and that these episodes were asymptomatic, leading to an underestimation of silent ischaemia.

The majority of episodes associated with no perceived trigger, or with mental stress alone, were silent and were accompanied by a low heart rate. The heart rate at onset of ischaemia appeared to parallel the symptoms of angina, with symptomatic episodes having a higher heart rate. The heart rate at onset of ischaemia 
also varied according to the type of stressor, being lowest for episodes associated with no stress or with mental stress alone, higher for episodes with physical stress, especially when vigorous, and highest for episodes associated with both physical and mental stress. This may explain why most of the episodes associated with both physical and mental stress provoked angina. These findings are consistent with a recent study which showed that a large proportion (approximately $80 \%$ ) of ambulatory ischaemic episodes are associated with periods of increased heart rate, and that the likelihood of developing angina is predicted by heart rate variables. ${ }^{18}$

It has been proposed that coronary vasoconstriction or abnormal vasomotor activity may explain episodes of ischaemia that are unrelated to increases in heart rate. ${ }^{13}{ }^{19}$ Mental stress has been shown to cause coronary vasoconstriction in patients with coronary disease in the catheterisation laboratory, ${ }^{19}$ and could be a cause of such ischaemia. In this study, the heart rate was low at the onset of episodes of daily life ischaemia in the absence of physical stress. Nevertheless, mental stress was perceived in a minority, and the cause of these episodes is unknown.

Combined physical and mental stress triggers usually provoked angina rather than silent ischaemia, and accounted for $15 \%$ of all anginal episodes. Examples of such stressors were "changing a car tyre and being anxious," and "hurrying to return the Holter monitor and being worried about being late." It is possible that autonomic overactivity with combined stressors may modulate both coronary tone and heart rate, as such episodes had the highest heart rate at the onset of ischaemia.

\section{LIMITATIONS}

Although we have interpreted the occurrence of any physical activity or possible mental stress at the time of ischaemia as a trigger, this may just represent an association rather than a causative factor or trigger. While the definition of both physical and mental stress was deliberately made very liberal to ensure high sensitivity, it is still possible that normal cerebral activity not perceived as stressful could have been responsible for the ischaemic episodes with no apparent trigger.
We thank Ms Diana Noyce and Dr Thomas Gumbrielle for their invaluable assistance in recruiting the patients, performing the study, and collecting the data. We thank Ms Phillipa Sobb and Ms Catherine Dobbin for their assistance in collection and analysis of the data, and $\mathrm{Mr}$ Connal Monro for his assistance in preparing the manuscript. The study was supported by a grant rom the National Heart Foundation of Australia, and QMED Inc provided the ambulatory electrographic recorders. Professor Ben Freedman was supported by the Medical Foundation of the University of Sydney, and Dr Cheuk Kit Wong by a Croucher Foundation Fellowship.

1 Schang S, Pepine C. Transient asymptomatic ST segment depression during daily activity. Am f Cardiol 1977;39:396402 .

2 Deanfield JE, Shea M, Kensett M, et al. Silent ischemia due to mental stress. Lancet 1984;ii:1001-5.

3 Nabel EG, Rocco MB, Barry J, et al. Asymtomatic ischemia in patients with coronary artery disease. $\mathscr{F} A M A$ 1987;257: 1923-8.

4 Cohn PF. Silent myocardial ischemia. Ann Intern Med 1988; 109:312-17.

5 Campbell S. Silent myocardial ischemia. A lot around but not easy to suspect. BMF 1988;297:751-2.

6 Mulcahy D, Keegan J, Crean P, et al. Silent myocardial ischemia in chronic stable angina: a study of its frequency and characteristics in 150 patients. Br Heart $\mathcal{F}$ 1988;60: 417-23.

7 Rozanski A, Bairey CN, Krantz DS, et al. Mental stress and the induction of silent myocardial ischemia in patients with coronary artery disease. $N$ Engl F Med 1988;318:1005-12.

8 Burg MM, Jain D, Soufer R, et al. Role of behavioral and psychological factors in mental-stress induced silent left ventricular dysfunction in coronary artery disease. $f \mathrm{Am}$ Coll Cardiol 1993;22:440-8.

9 Deanfield J, Shea M, Wislon R, et al. Direct effects of smoking on the heart: silent ischemic disturbances of coronary flow. Am ₹ Cardiol 1986;57:1005-9.

10 Barry J, Mead K, Nabel EG, et al. Effect of smoking on the activity of ischemic heart disease. $7 A M A$ 1989;261:398402 .

11 Deanfield J, Shea M, Ribiero P, et al. Transient ST-segment depression as a marker of myocardial ischemia during daily life. Am f Cardiol 1984;54:1195-200.

12 Cecchi A, Dovellini E, Archi F, et al. Silent myocardial ischemia during ambulatory electrocardiographic monitoring in patients with effort angina. $\mathcal{F}$ Am Coll Cardiol 1983;1: 934-9.

13 Gottdiener JS, Krantz DS, Howell RH, et al. Induction of silent myocardial ischemia with mental stress testing: relation to the triggers of ischemia during daily life activities and to ischemic functional severity. 7 Am Coll Cardiol 1994; and to ischemic

14 Wong CK, Freedman SB. Usefulness of laboratory mental stress test in patients with stable coronary artery disease. Clin Cardiol 1997;20:367-71.

15 Barry J, Selwyn AP, Nabel EG, et al. Frequency of ST-segment depression produced by mental stress in stable angina pectoris from coronary artery disease. $\mathrm{Am} \mathcal{F}$ Cardiol 1988;61:989-93.

16 Gullette ECD, Blumenthal JA, Babyak M, et al. Effects of mental stress on myocardial ischemia during daily life. 7AMA 1997;277:1521-6.

17 Jamal SM, Mitra-Duncan L, Kelly DT, et al. Validation of a real-time electrocardiographic monitor for detection of myocardial ischemia secondary to coronary artery disease. Am f Cardiol 1987;60:525-7.

18 Andrews TC, Fenton T, Toyosaki N, et al. Subsets of ambulatory myocardial ischemia based on heart rate variability: circadian distribution and response to anti-ischemic medications. Circulation 1993;88:92-100.

19 Yeung AC, Vekshtein VI, Krantz DS, et al. The effect of atherosclerosis on the vasomotor response of coronary arteries to mental stress. $N$ Engl F Med 1991;325:1551-6. 